\title{
GROWTH OF HP FUNCTIONS IN TUBES
}

\author{
RICHARD D. CARMICHAEL AND STEPHEN P. RICHTERS \\ Department of Mathematics, Wake Forest University \\ Winston-Salem, North Carolina 27109 U.S.A. \\ (Received October 9, 1980)
}

ABSTRACT. Let $C$ be an open convex cone in $n$ dimensional real space $R^{n}$ such that $\bar{C}$ does not contain any entire straight line. We obtain a growth condition on functions in the Hardy spaces $\mathrm{I}_{\mathrm{P}}^{\mathrm{P}}\left(\mathrm{T}^{\mathrm{C}}\right), 1 \leq \mathrm{p}<\infty$, corresponding to the tube $\mathrm{T}^{\mathrm{C}}=\mathrm{R}^{\mathrm{n}}+\mathrm{iC}$ in $\mathrm{n}$ dimensional complex space $\mathbb{C}^{\mathrm{n}}$.

KEY WORDS AND PHRASES. $H^{P}$ Spaces in Tube Domains, Poisson Kernel Function and Poisson Integral.

1980 MATHEMATICS SUBJECT CLASSIFICATION CODES. 32A07, 32A10, 32A25, $32 A 35$.

\section{INTRODUCTION.}

Throughout this paper $\langle\cdot, \cdot\rangle$ denotes the inner product of points in $R^{n}$ or $\mathbb{C}^{\mathrm{n}}$; and $\overline{0}$ denotes the origin in $\mathrm{R}^{\mathrm{n}}$, that is the $\mathrm{n}$-tuple of zeros. A set $C \subset \mathrm{R}^{\mathrm{n}}$ is a cone (with vertex at the origin $\overline{0}$ in $\mathrm{R}^{\mathrm{n}}$ ) if $\mathrm{y} \varepsilon \mathrm{C}$ implies $\lambda y \varepsilon C$ for all points y $\varepsilon C$ and all positive scalars $\lambda$. The intersection of $C$ with the unit sphere $|\mathrm{y}|=1$ in $\mathrm{R}^{\mathrm{n}}$ is called the projection of the cone $\mathrm{C}$ and is denoted $\operatorname{pr}(\mathrm{C})$. A cone $C^{\prime}$ such that $\operatorname{pr}\left(\overline{C^{\prime}}\right) \subset \operatorname{pr}(C)$ will be called a compact subcone of the cone C. The dual cone $C^{*}$ of a cone $C$ is defined as $C^{*}=\left\{t \varepsilon R^{n}:\langle t, y\rangle \geq 0\right.$ for all y $\varepsilon$ C $\}$. See [1, p. 218] for these definitions and further relevant facts concerning cones.

Let $\mathrm{C}$ be an open convex cone such that $\overline{\mathrm{C}}$ does not contain any entire straight 
line. Then $C^{*}$ has a nonvoid interior [1, p. 222, Lemma 1 and line 15] in $R^{n}$. (Hence $C$ is a regular cone in the sense of $[2, p .275]$ and [3, p. 101].) This fact together with the first conclusion of [4, Lemma 1] and a computation as in $[4,(6)]$ show that for each y $\varepsilon C$ and any $p>0$ there is a $\delta=\delta_{y}>0$ depending on y $\varepsilon C$ such that

$$
0<\int_{C^{*}} e^{-p<y, n>} d n \leq \int_{C^{*}} e^{-p \delta|y||n|} d n \leq \Omega_{n}(n-1) !(p \delta|y|)^{-n}<\infty
$$

for any cone C satisfying the above assumptions, a fact which is important for this paper. Here $\Omega_{n}$ is the surface area of the unit sphere in $R^{n}$. (Note that the word "volume" in [4, p. 577, 1ines 11 and 18] should be replaced by "surface area".)

Let the cone $\mathrm{C}$ satisfy the assumptions of the preceding paragraph. The Cauchy kernel function corresponding to the tube $T^{C}=R^{n}+i C$ is $([5, p .201],[6])$

$$
K(z-t)=\int_{C^{*}} e^{2 \pi i<z-t, n>} d n, z \in T^{C}, t \in R^{n} .
$$

The Poisson kernel function corresponding to $T^{C}$ is $([5$, p. 204, (18) $],[6])$

$$
Q(z ; t)=\frac{K(z-t) \overline{K(z-t)}}{K(2 i y)}=\frac{|K(z-t)|^{2}}{K(2 i y)}, z=x+i y \varepsilon T^{C}, t \varepsilon R^{n} .
$$

Both $K(z-t)$ and $Q(z: t)$ are well defined and satisfy various properties ([5], [6].) For our purposes here we note that $Q(z ; t)$ is an approximate identity ([2, Prop. 2], [5, Lemma 6]) and satisfies

$$
0 \leq Q(z ; t)=\frac{|K(z-t)|^{2}}{K(2 i y)} \leq \frac{(K(i y))^{2}}{K(2 i y)}, z=x+i y \varepsilon T^{C}, t \varepsilon R^{n},
$$

with the second inequality following directly from the definition of $K(z-t)$.

The Hardy spaces $H^{p}\left(T^{C}\right), 0<p \leq \infty$, corresponding to a tube $T^{C}=R^{n}+i C$ in $\mathbb{C}^{\mathrm{n}}$ are defined in [2] and [3, pp. $\left.90-91\right]$. Note that $\mathrm{H}^{\infty}\left(\mathrm{T}^{\mathrm{C}}\right)$ is the space of all bounded holomorphic functions in $\mathrm{T}^{\mathrm{C}}$.

Let $C_{1}=\left\{y \in R^{n}: y_{j}>0, j=1, \ldots, n\right\}$. Madych [7] has proved that if $f(z) \varepsilon$ ${ }_{\mathrm{H}}^{\mathrm{p}}\left(\mathrm{T}^{\mathrm{C}}{ }^{1}\right), 0<\mathrm{p}<\infty$, then there exists a constant $\mathrm{M}(\mathrm{f})$ depending on $\mathrm{f}$ such that 


$$
|f(x+i y)| \leq M(f)\left(\prod_{j=1}^{n} y_{j}\right)^{-1 / p}, z=x+i y \varepsilon T^{C_{1}} .
$$

In this note we prove a growth result for functions $f(z) \varepsilon H^{p}\left(T^{C}\right)$ for certain values of $p$ and for a general class of cones C. Our result is as follows.

THEOREM. Let $C$ be any open convex cone in $R^{n}$ such that $\bar{C}$ does not contain any entire straight line. Let $\mathrm{f}(\mathrm{z}) \in \mathrm{H}^{\mathrm{p}}\left(\mathrm{T}^{\mathrm{C}}\right), 1 \leq \mathrm{p}<\infty$. For any compact subcone $C^{\prime}$ of $C$ there exists a constant $M\left(f ; C^{\prime}\right)$ depending on $f$ and on $C^{\prime}$ such that

$$
|f(x+i y)| \leq M\left(f ; C^{\prime}\right)|y|^{-n / p}, z=x+i y \varepsilon T^{C^{\prime}} .
$$

In the special case of the THEOREM that $C=C_{1}$, liadych's growth (1.2) is better than (1.3) in that (1.2) holds for all points in the tube $\mathrm{T}^{\mathrm{C}}{ }_{1}$. Of course a special case of the result $(1.2)$ is if $n=1$ in which case $T^{C_{1}}$ is the upper half plane in $\mathbb{C}^{1}$; and the growth (1.2) holds also for the lower half plane in $\mathbb{C}^{1}$ with y replaced by $|y|$ in (1.2). For arbitrary cones satisfying the hypotheses of the THEOREM we are able to obtain (1.3) holding for all $z \varepsilon \mathrm{T}^{\mathrm{C}^{\prime}}$, $\mathrm{C}^{\prime}$ being an arbitrary compact subcone of $\mathrm{C}$; and our THEOREM has interest for dimension $\mathrm{n} \geq 2$ since the growth of $\mathrm{H}^{\mathrm{P}}$ functions corresponding to the upper and lower half planes in $\mathbb{C}^{1}$ is already known as noted above. Note that at least for the case $p=2$, the hypothesis on $\bar{C}$ in the THEOREM is needed to ensure that $\mathrm{H}^{2}\left(\mathrm{~T}^{\mathrm{C}}\right)$ contains a function that is not identically zero on $\mathrm{T}^{\mathrm{C}}[3, \mathrm{p} .94$, Corollary 2.6], and we further need this assumption for all $p, 1 \leq p<\infty$, in order to have $Q(z ; t)$ well defined in our proof of the THEOREM which is given in section 2 of this paper. Because of [3, p. 93, Corollary 2.4] there is no loss of generality in letting the cone $\mathrm{C}$ be convex in the THEOREM for $\mathrm{p}=2$; we assume $\mathrm{C}$ is convex for the other $\mathrm{p}$ also for otherwise we would prove the THEOREM for $\mathrm{H}^{\mathrm{p}}\left(\mathrm{T}^{\mathrm{O}(\mathrm{C})}\right), \mathrm{O}(\mathrm{C})$ being the convex envelope of the assumed open and connected cone $C . H^{\infty}\left(T^{C}\right)$ is by definition the space of all bounded holomorphic functions in $\mathrm{T}^{\mathrm{C}}$; hence we did not include the case $\mathrm{p}=\infty$ in the THEOREM since the growth of $\mathrm{H}^{\infty}\left(\mathrm{T}^{\mathrm{C}}\right)$ functions is known by definition.

In [4] we obtained the THEOREM for the case $p=2$ using analysis involving 
the Cauchy integral representation of functions in $\mathrm{H}^{2}\left(\mathrm{~T}^{\mathrm{C}}\right)$. In this note we are able to extend this growth result to the spaces $\mathrm{H}^{\mathrm{p}}\left(\mathrm{T}^{\mathrm{C}}\right), 1 \leq \mathrm{p}<\infty$, using the Poisson integral representation of functions in $\mathrm{H}^{\mathrm{p}}\left(\mathrm{T}^{\mathrm{C}}\right)$. The growth (1.3) is of independent interest, but we are especially interested in it because it aids us in analysis relating certain spaces of holomorphic functions in tubes having distributional boundary values with $\mathrm{H}^{\mathrm{p}}$ spaces in tubes, a topic of research which has proved useful in mathematical physics and which we are presently pursuing.

\section{PROOF OF THE THEOREM.}

We prove the THEOREM with the aid of two lemmas. Throughout this section $\beta_{u, v}$ denotes the angle between the two nonzero vectors $u \varepsilon R^{n}$ and $v \varepsilon R^{n}$ with this angle being defined in the usual way.

LEMMA 1. Let the cone $C$ be as in the THEOREM. Let $C^{\prime}$ be an arbitrary compact subcone of $C$. There exists a constant $A\left(C^{\prime}\right)$ depending on $C^{\prime}$ such that

$$
K(i y) \leq A\left(C^{\prime}\right)|y|^{-n}, y \in C^{\prime} \text {. }
$$

We give two proofs of Lemma 1.

FIRST PROOF OF LEMMA 1. For any compact subcone $C^{\prime}$ of $C$ choose an angle $\gamma_{0}$ depending on $C^{\prime}$ such that $0<\gamma_{0}<\pi / 4$ and

$$
\gamma_{0}<\inf \left\{\beta_{u, v}: u \varepsilon \partial C \cap \operatorname{pr}(C), v \varepsilon \partial C^{\prime} \cap \operatorname{pr}\left(C^{\prime}\right)\right\}
$$

where $\partial C\left(\partial C^{\prime}\right)$ denotes the boundary of $C\left(C^{\prime}\right)$; and $\gamma_{0}$ exists. Let y $\varepsilon C^{\prime}$ be arbitrary and put

$$
\Gamma_{y, \gamma_{0}}=\left\{\eta \varepsilon R^{n}: \eta \neq \overline{0}, 0 \leq \beta_{n, y}<\gamma_{0}\right\} .
$$

Then $y \in \Gamma_{y, \gamma_{0}}, \Gamma_{y, \gamma_{0}}$ is an open convex cone in $R^{n}$, and $\Gamma_{y, \gamma_{0}} \subset \subset$ because of the choice of $\gamma_{0}$; hence the corresponding dual cones satisfy $C^{*} \subseteq \Gamma_{y, \gamma_{0}}^{*}$. Thus for the $y \in C^{\prime} \subset C$

$$
K(\text { iy })=\int_{C^{*}} e^{-2 \pi<y, n>} d n \leq \int_{\Gamma_{y, \gamma_{0}}^{*}} e^{-2 \pi<y, n>} d n=\int_{\Gamma_{y, \gamma_{0}}^{*} \backslash\{\overline{0}\}} e^{-2 \pi<y, n>} d n .
$$

(The first equality in (2.2) is the definition of the Cauchy kernel K(iy), y $\varepsilon$ C.) 
We switch to $\mathrm{n}$ dimensional spherical coordinates ([8, pp. $217-218]$, [9]) by letting

$$
n=r \Psi
$$

where $\mathrm{r}=|n|$ and

$\Psi=$

$\left(\cos \left(\theta_{1}\right), \sin \left(\theta_{1}\right) \cos \left(\theta_{2}\right), \ldots, \sin \left(\theta_{1}\right) \ldots \sin \left(\theta_{n-2}\right) \cos \left(\theta_{n-1}\right), \sin \left(\theta_{1}\right) \ldots \sin \left(\theta_{n-2}\right) \sin \left(\theta_{n-1}\right)\right)$

with $\mathrm{n}$ being the dimension; and

$$
\begin{aligned}
& 0 \leq \theta_{j} \leq \pi, j=1, \ldots, n-2, \\
& 0 \leq \theta_{n-1}<2 \pi .
\end{aligned}
$$

Put

$$
\Psi^{\prime}=\left\{\left(\theta_{1}, \theta_{2}, \ldots, \theta_{n-1}\right): \eta=r \Psi \varepsilon \Gamma_{y, \gamma_{0}}^{*} \backslash\{\overline{0}\}, 0<r<\infty\right\} .
$$

Noting the Jacobian of the transformation (2.3) in $[8, p .218]$ we obtain from (2.2) that

$$
\begin{aligned}
& \text { K(iy) } \leq \int_{\Gamma_{y, \gamma_{0}}^{*} \backslash\{\overline{0}\}} e^{-2 \pi<y, n>} d n= \\
& =\int_{\Psi^{\prime}} \int_{0}^{\infty} r^{n-1} e^{-2 \pi r<y, \Psi>} \sin ^{n-2}\left(\theta_{1}\right) \sin ^{n-3}\left(\theta_{2}\right) \ldots \sin \left(\theta_{n-2}\right) d r d \theta_{1} \ldots d \theta_{n-1}
\end{aligned}
$$

for $y \varepsilon C^{\prime} \subset C$. It follows from the definition of $\Gamma_{y, \gamma_{0}}$ that for any $\eta \varepsilon \Gamma_{y, \gamma_{0}}^{*} \backslash\{\overline{0}\}$

$$
0 \leq \beta_{n, y} \leq(\pi / 2)-\gamma_{0}<\pi / 2
$$

so that $r\langle y, \Psi\rangle=\langle y, \eta\rangle=|y||\eta| \cos \left(\beta_{\eta, y}\right)>0$ in the right side of (2.6). Thus integration by parts $(n-1)$ times on the improper integral with respect to $r$ in (2.6) yields

$$
\int_{0}^{\infty} r^{n-1} e^{-2 \pi r<y, \Psi>} d r=\frac{(n-1) !}{(2 \pi<y, \Psi>)^{n}} .
$$

The $\Psi$ defined in (2.4) satisfies $|\Psi|=1$. As $\eta=r \Psi$ varies over $\Gamma_{y, \gamma_{0}}^{*} \backslash\{\overline{0}\}, \Psi$ varies over $\operatorname{pr}\left(\Gamma_{\mathrm{y}, \gamma_{\mathrm{o}}}^{*}\right)$; and from $(2.7), 0 \leq \beta_{\Psi, \mathrm{y}} \leq(\pi / 2)-\gamma_{\mathrm{o}}<\pi / 2$ so that 
$\cos \left(\beta_{\Psi, y}\right) \geq \cos \left((\pi / 2)-\gamma_{0}\right)>0$. Thus $\langle y, \psi\rangle$ in the right side of $(2.6)$, and hence in $(2.8)$, satisfies

$$
\langle y, \Psi\rangle=|y||\Psi| \cos \left(\beta_{\Psi, y}\right)=|y| \cos \left(\beta_{\Psi, y}\right) \geq|y| \cos \left((\pi / 2)-\gamma_{0}\right)>0 .
$$

Using (2.9) and (2.8) in (2.6), we have

$K(i y) \leq \frac{(n-1) !}{\left(2 \pi|y| \cos \left((\pi / 2)-\gamma_{0}\right)\right)^{n}} \int_{\Psi^{\prime}} \sin ^{n-2}\left(\theta_{1}\right) \sin ^{n-3}\left(\theta_{2}\right) \ldots \sin \left(\theta_{n-2}\right) d \theta_{1} \ldots d \theta_{n-1}$ for $y \varepsilon C^{\prime} \subset C$. From (2.5) we see that the integrand in (2.10) is a nonnegative function over $\Psi^{\prime}$ and indeed over the whole of $\Psi^{\prime \prime}=\left\{\left(\theta_{1}, \theta_{2}, \ldots, \theta_{n-1}\right): 0 \leq \theta_{j} \leq \pi\right.$, $\left.\mathrm{j}=1, \ldots, \mathrm{n}-2,0 \leq \theta_{\mathrm{n}-1}<2 \pi\right\}$; and the integral in (2.10) is a finite positive real number. Thus we replace $\Psi^{\prime}$ in $(2.10)$ by $\Psi^{\prime \prime}$ and obtain

$$
K(i y) \leq A\left(C^{\prime}\right)|y|^{-n}
$$

where

$A\left(C^{\prime}\right)=\frac{(n-1) !}{\left(2 \pi \cos \left((\pi / 2)-\gamma_{0}\right)\right)^{n}} \int_{\Psi^{\prime},} \sin ^{n-2}\left(\theta_{1}\right) \sin ^{n-3}\left(\theta_{2}\right) \ldots \sin \left(\theta_{n-2}\right) d \theta_{1} \ldots d \theta_{n-1}$, and the integral on the right is a finite positive real number. This constant $A\left(C^{\prime}\right)$ depends on $C^{\prime}$ since the choice of the angle $\gamma_{0}$ at the beginning of the proof depends on $C^{\prime}$. However, $A\left(C^{\prime}\right)$ does not depend on y $\varepsilon C^{\prime} ;(2.11)$ holds for all y $\varepsilon C^{\prime}$ with $C^{\prime}$ being any compact subcone of $C$. Thus $(2.1)$ is obtained, and the first proof of Lemma 1 is complete.

SECOND PROOF OF LEMMA 1. Let $C^{\prime}$ be an arbitrary compact subcone contained in C. By the second conclusion of [4, Lemma 1] there exists a $\delta=\delta\left(C^{\prime}\right)>0$ depending only on $C^{\prime}$ such that $\langle y, n\rangle \geq \delta|y||n|$ for all y $\varepsilon C^{\prime}$ and all $\eta \varepsilon C^{*}$. This inequality and the same calculation as in $[4,(6)]$ yield

$$
\begin{aligned}
\mathrm{K}(\mathrm{iy}) & =\int_{\mathrm{C}^{*}} \mathrm{e}^{-2 \pi<y, n>} \mathrm{d} \eta \leq \int_{\mathrm{C}^{*}} \mathrm{e}^{-2 \pi \delta|y||n|} \mathrm{d} n \leq \int_{\mathrm{R}^{n}} \mathrm{e}^{-2 \pi \delta|\mathrm{y}||n|} \mathrm{dn}= \\
& =\Omega_{\mathrm{n}} \int_{0}^{\infty} \mathrm{r}^{\mathrm{n}-1} \mathrm{e}^{-2 \pi \delta|\mathrm{y}| \mathrm{r}} \mathrm{dr}=\Omega_{\mathrm{n}}(\mathrm{n}-1) !(2 \pi \delta|\mathrm{y}|)^{-\mathrm{n}}
\end{aligned}
$$

for all y $\varepsilon C^{\prime} \subset C$, where $\Omega_{n}$ is the surface area of the unit sphere in $R^{n}$. (As 
noted in the second paragraph of the Introduction, the word "volume" in [4, p. 577, lines 11 and 18] should be replaced by "surface area".) Thus (2.1) is again proved with

$$
A\left(C^{\prime}\right)=\Omega_{n}(n-1) !(2 \pi \delta)^{-n}
$$

depending on $C^{\prime} \subset C$ since $\delta$ depends on $C^{\prime}$. The second proof of Lemma 1 is complete.

LEMMA 2. Let the cone $C$ be as in the THEOREM. There exists a constant $B(C)$ depending on $C$ such that

$$
\mathrm{K}(2 \mathrm{iy}) \geq \mathrm{B}(\mathrm{C})|\mathrm{y}|^{-\mathrm{n}}, \mathrm{y} \in \mathrm{C} .
$$

PROOF. By the first conclusion of [4, Lemma 1] we have that $\langle y, n\rangle\rangle 0$ for all y $\varepsilon C$ and all $\eta \varepsilon C^{*} \backslash\{\overline{0}\}$, which is a nonvoid set by the discussion in the second paragraph of the Introduction, under the assumptions on the cone C. Using this fact, a change to spherical coordinates in $n$ dimensions as in the first proof of Lemma 1, and a computation as in (2.8), we obtain for all y $\in \mathrm{C}$ that

$$
\begin{aligned}
& K(2 i y)=\int_{C^{*}} e^{-4 \pi<y, n>} d n=\int_{C^{*}} e_{\{\overline{0}\}} e^{-4 \pi<y, n>} d n= \\
& =\frac{(n-1) !}{(4 \pi)^{n}} \int_{\Phi}\left(\langle y, \Psi>)^{-n} \sin ^{n-2}\left(\theta_{1}\right) \sin ^{n-3}\left(\theta_{2}\right) \ldots \sin \left(\theta_{n-2}\right) d \theta_{1} \ldots d \theta_{n-1}\right.
\end{aligned}
$$

where $\Psi$ is defined in $(2.4)$ and $\Phi=\left\{\left(\theta_{1}, \theta_{2}, \ldots, \theta_{n-1}\right): n=r \Psi \varepsilon C^{*} \backslash\{\overline{0}\}, 0<r<\infty\right\}$. In (2.13) $\Psi \varepsilon \operatorname{pr}\left(C^{*}\right)$ and $\langle y, \Psi\rangle>0$ for all y $\varepsilon$ C. Thus for $\langle y, \Psi\rangle$ in (2.13)

$$
0<\langle y, \Psi\rangle=|y||\Psi| \cos \left(\beta_{y, \Psi}\right) \leq|y|, y \varepsilon C .
$$

Using (2.14) in (2.13) we get for any y $\varepsilon$ C that

$$
K(2 i y) \geq \frac{(n-1) !}{(4 \pi|y|)^{n}} \int_{\Phi} \sin ^{n-2}\left(\theta_{1}\right) \sin ^{n-3}\left(\theta_{2}\right) \ldots \sin \left(\theta_{n-2}\right) d \theta_{1} \ldots d \theta_{n-1} .
$$

The integral on the right of (2.15) is a finite positive real number, and (2.15) holds for all y $\varepsilon$ C. Further the integral in (2.15) depends only on the cone $C$ since $\Phi$ depends only on $C^{*}$ which depends only on $C$. The integral on the right of (2.15) is independent of $y \in C .(2.15)$ is thus the desired inequality (2.12) with

$$
B(C)=(n-1) !(4 \pi)^{-n} \int_{\Phi} \sin ^{n-2}\left(\theta_{1}\right) \sin ^{n-3}\left(\theta_{2}\right) \ldots \sin \left(\theta_{n-2}\right) d \theta_{1} \ldots d \theta_{n-1} .
$$

The proof of Lemma 2 is complete. 
PROOF OF THE THEOREM. By [2, Prop. 4], given $\mathrm{f}(\mathrm{z}) \in \mathrm{H}^{\mathrm{P}}\left(\mathrm{T}^{\mathrm{C}}\right), 1 \leq \mathrm{p}<\infty$, there is a function $h \in L^{p}\left(R^{n}\right)$ such that

$$
f(z)=\int_{R^{n}} h(t) Q(z ; t) d t, z \varepsilon T^{C} .
$$

Using this equality, the fact that the Poisson kernel $Q(z ; t), z \in T^{C}, t \in R^{n}$, is an approximate identity ([2, Prop. 2], [5, Lemma 6]), Jensen's inequality $[10$, p. $91,2.4 .19]$, and $(1.1)$, we obtain for $z=x+i y \in T^{C}$ that

$$
\begin{aligned}
|f(x+i y)|^{P} & =\left|\int_{R^{n}} h(t) Q(z ; t) d t\right|^{P} \leq \\
& \leq \int_{R^{n}}|h(t)|^{P} Q(z ; t) d t \leq \frac{(K(i y))^{2}}{K(2 i y)} \int_{R^{n}}|h(t)|^{P} d t .
\end{aligned}
$$

Now let $C^{\prime}$ be an arbitrary compact subcone of $C$. Using Lemmas 1 and 2 in (2.16) we obtain constants $A\left(C^{\prime}\right)$ and $B(C)$ such that

$$
|f(x+i y)|^{p} \leq \frac{\left(A\left(C^{\prime}\right)\right)^{2}}{B(C)}|y|^{-n} \int_{R} n|h(t)|^{p} d t, z=x+i y \varepsilon T^{C^{\prime}} .
$$

The desired growth (1.3) with

$$
M\left(f ; C^{\prime}\right)=\left(\left(A\left(C^{\prime}\right)\right)^{2} / B(C)\right)^{1 / p}\|h\|_{L} p
$$

now follows by taking the pth. root of both sides of (2.17). The proof of the THEOREM is complete.

\section{REFERENCES}

1. VLADIMIROV, V. S. Methods of the Theory of Functions of Many Complex Variables, M. I. T. Press, Cambridge, Mass., 1966.

2. KORÁNYI, A. A Poisson Integral for Homogeneous Wedge Domains, J. Analyse Math. 14 (1965) $275-284$.

3. STEIN, E. M. and G. WEISS. Introduction to Fourier Analysis on Euclidean Spaces, Princeton University Press, Princeton, N. J., 1971.

4. CARMICHAEL, Richard D. and Elmer K. HAYASHI. A Pointwise Growth Estimate for Analytic Functions in Tubes, Internat. J. Math. Math. Sci. 3 (1980) $575-581$.

5. CARMICHAEL, Richard D. Generalized Cauchy and Poisson Integrals and Distributional Boundary Values, SIAM J. Math. Anal. 4 (1973) $198-219$. 
6. CARMICHAEL, Richard D. and Elmer K. HAYASHI. Analytic Functions in Tubes Which Are Representable by Fourier-Laplace Integrals, Pacific J. Math. $\underline{90}$ (1980) $51-61$.

7. MADYCH, W. R. Distributions with Strong Maximal Functions in $L^{\mathrm{P}}\left(\mathrm{R}^{\mathrm{n}}\right)$, preprint.

8. FLEMING, Wendell. Functions of Several Variables, 2nd Edition, Springer-Verlag, New York, 1977.

9. BLUMENSON, L. E. A Derivation of n-dimensional Spherical Coordinates, Amer. Math. Monthly 67 (1960) $63-66$.

10. FEDERER, Herbert. Geometric Measure Theory, Springer-Verlag, New York, 1969. 


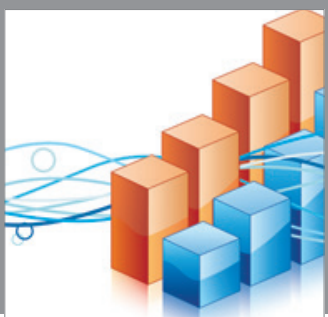

Advances in

Operations Research

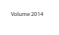

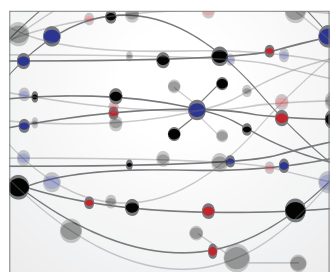

\section{The Scientific} World Journal
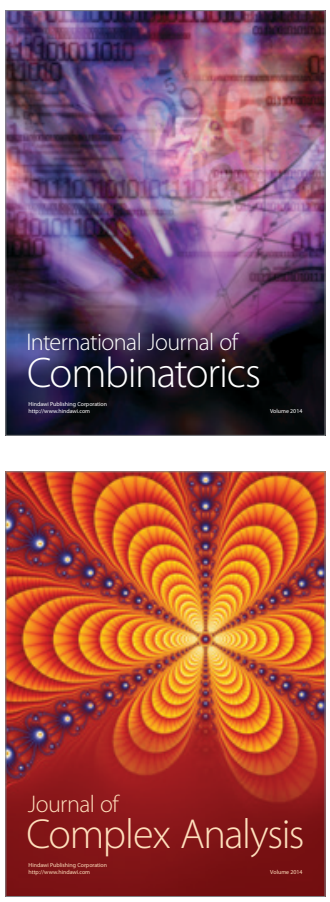

International Journal of

Mathematics and

Mathematical

Sciences
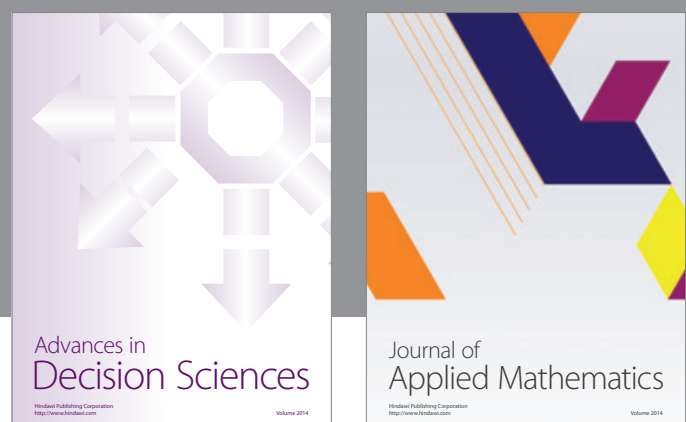

Journal of

Applied Mathematics
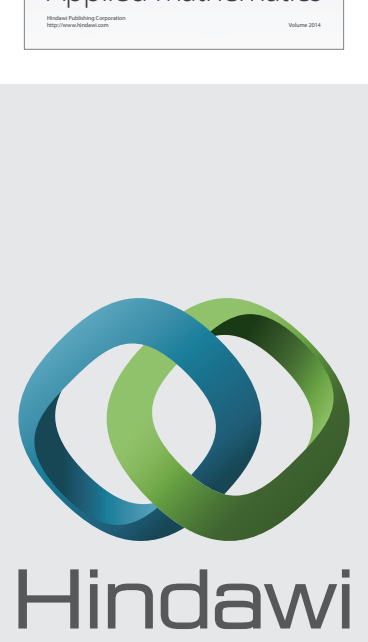

Submit your manuscripts at http://www.hindawi.com
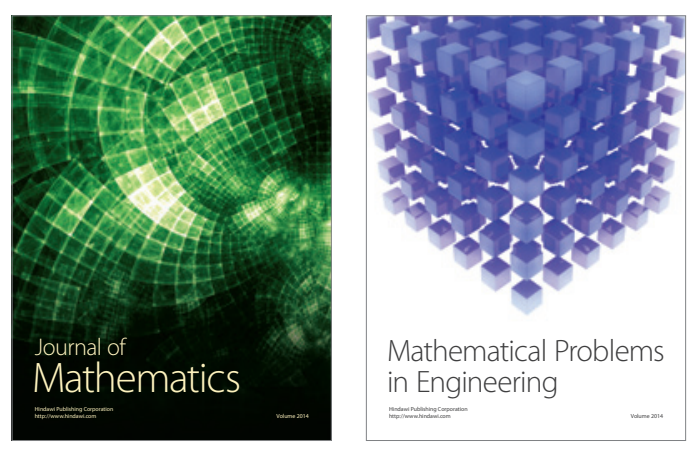

Mathematical Problems in Engineering
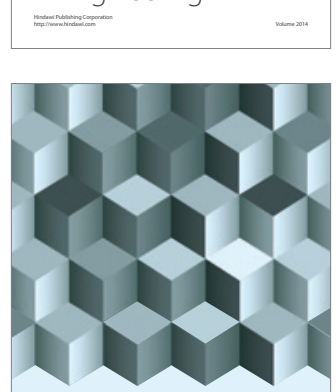

Journal of

Function Spaces
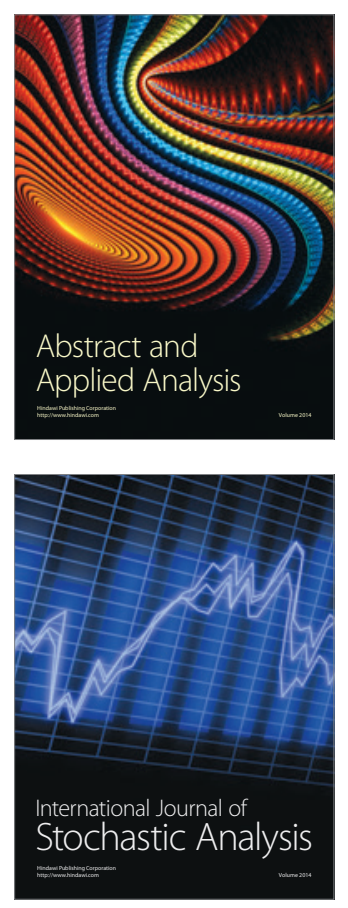

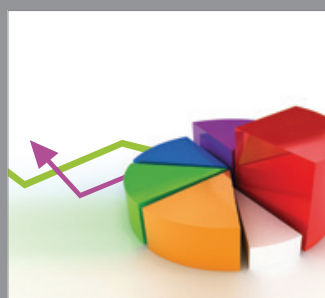

ournal of

Probability and Statistics

Promensencen
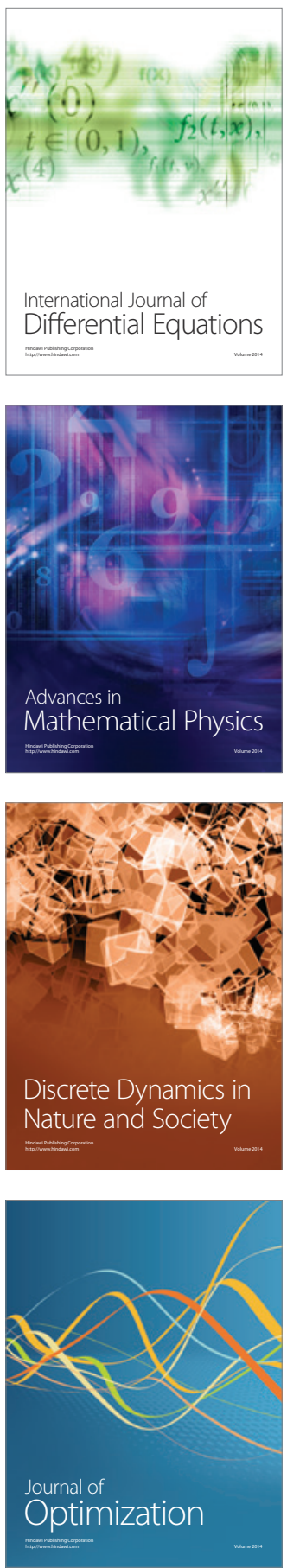